\title{
Die Therapeutisierung des Sozialen
}

\section{Einleitung in den Schwerpunkt}

Mit dem Thema "Therapeutisierung des Sozialen" greifen wir in diesem Schwerpunkt einen theoretischen Diskurs wieder auf, der in den 1970er und 1980er Jahre unter den Begriffen Psychiatrisierung und Therapeutisierung des Alltags Bestandteil kritischer Gesellschaftsanalyse war. Ein wesentlicher Motor war die damalige vehemente Kritik an den biologistisch-medizinisch ausgerichteten psychiatrischen Denk-, Handlungs- und Beziehungsmuster, einschließlich des omnipotenten Anspruchs ihrer professionellen Protagonist_innen auf Alleinzuständigkeit und Deutungshoheit für psychosoziale Phänomene, die als psychische Auffälligkeiten oder Erkrankungen ins Gesundheitssystem verschoben wurden.

$\mathrm{I}$ m Zuge dieser Kritik wurde insgesamt die Geltung der als repressiv und diskriminierend empfundenen Normen der Mehrheitsgesellschaft radikal hinterfragt. Statt gesellschaftliche Strukturen sowie Machtund Produktionsverhältnisse im Anschluss an soziologische Diskurse zu thematisieren, wie etwa den der Anomietheorie (vgl. Durkheim 1973, 1992; Merton 1968; Böhnisch 1994), werde die Identifizierung, Behandlung und Kontrolle von „schwierigen“ Menschen mithilfe einer psychiatrisch-medizinischen Perspektive legitimiert, um sie nach den Erfordernissen der kapitalistischen Gesellschaft an die jeweils vorherrschenden Bedingungen und Normen anzupassen. Aus diesen Gründen bezeichneten Basaglia und Basaglia-Ongaro (1980) die gesellschaftliche Funktion der damit befassten Professionellen als „Befriedungsverbrecher“. Frau-

\section{Margret Dörr
Katholische Hochschule Mainz, Mainz, Deutschland
*1956, Dr., Professorin an der Katholischen Hochschule
Mainz, Fachbereich Soziale Arbeit und Sozialwissenschaften.
$\begin{aligned} & \text { Arbeitsschwerpunkte: Theorien Sozialer Arbeit; Biographie und } \\ & \text { Sozialisationstheorie, Psychoanalytische (Sozial-) Pädagogik. } \\ & \text { margret.doerr@kh-mz.de }\end{aligned}$}

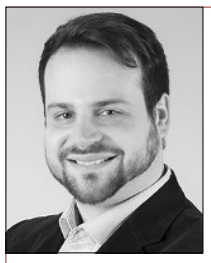

\section{Dirk Kratz}

Landau, Deutschland

*1980, Dr. phil., Diplom-Pädagoge, seit 2015 Geschäftsführer des Therapieverbundes Ludwigsmühle in Rheinland-Pfalz mit dem Arbeitsschwerpunkt soziale Rehabilitation und Inklusion in der Suchthilfe.

dirk.kratz@ludwigsmuehle.de

Schlüsselwörter Therapeutisierung, Psychiatrisierung, Stigmatisierung, Optimierung enbewegung, Selbsthilfegruppen, Friedensbewegung etc. wiesen gleichzeitig auf eine andere Perspektive, durch die der therapeutische Diskurs in den 1960 bis 80er Jahren als Auseinandersetzung mit den eigenen Gefühlen politisch gewendet und emphatisch als emanzipatorischer Akt und Teil eines Projekts der Demokratisierung begriffen wurde (vgl. Maasen 2011; Hitzer 2011).

Im aktuellen Psychiatrisierungs- und Therapeutisierungsdiskurs werden die sozio-ökonomischen und politisch-kulturellen Ungleichheits- und Konfliktverhältnisse weiterhin weitgehend ausgeblendet. Zugleich zielt er aber, der Individualisierungsthese folgend, auf eine zunehmende Eigenaktivität im Sinne individueller Selbstverantwortung und Selbstsorge oder kurz: auf eine optimierte Selbstführung des Subjektes. Mangelnde Selbstsorge und fehlende Aktivität wird nunmehr als ein „Ausweis individueller Unfähigkeit oder persönlichen Unwillens" (Lessenich 2008, S. 83) begriffen. Dadurch scheinen „[d]ie endlose Arbeit am Ich [...] und Selbstprüfung, die ihrerseits eine Subjektivierungs- und Herrschaftstechnik darstellt" (Han 2014, S. 44), sowie die Vision einer bio-psycho-sozial optimierten Selbstführung inzwischen zu selbstverständlichen Bestandteilen des gesellschaftlichen Alltags geworden zu sein. Viele Bürger_innen teilen beispielsweise eine Kritik an der Beschleunigung und Verdichtung des Alltags, der zu dauerhaftem Stress führe, und an den so gestiegenen Bewältigungsanforderungen für die/den Einzelne_n. Sie fühlen sich einerseits angesprochen durch Konsumangebote wie Beratungs- und Coaching-Literatur, Anti-Stress-Seminare oder auch Wellness- und Wellbeing-Tourismus, deren Annahme als Ausdruck verantwortlicher Selbstsorge und eines Bemühens um eigene Weiterentwick- 


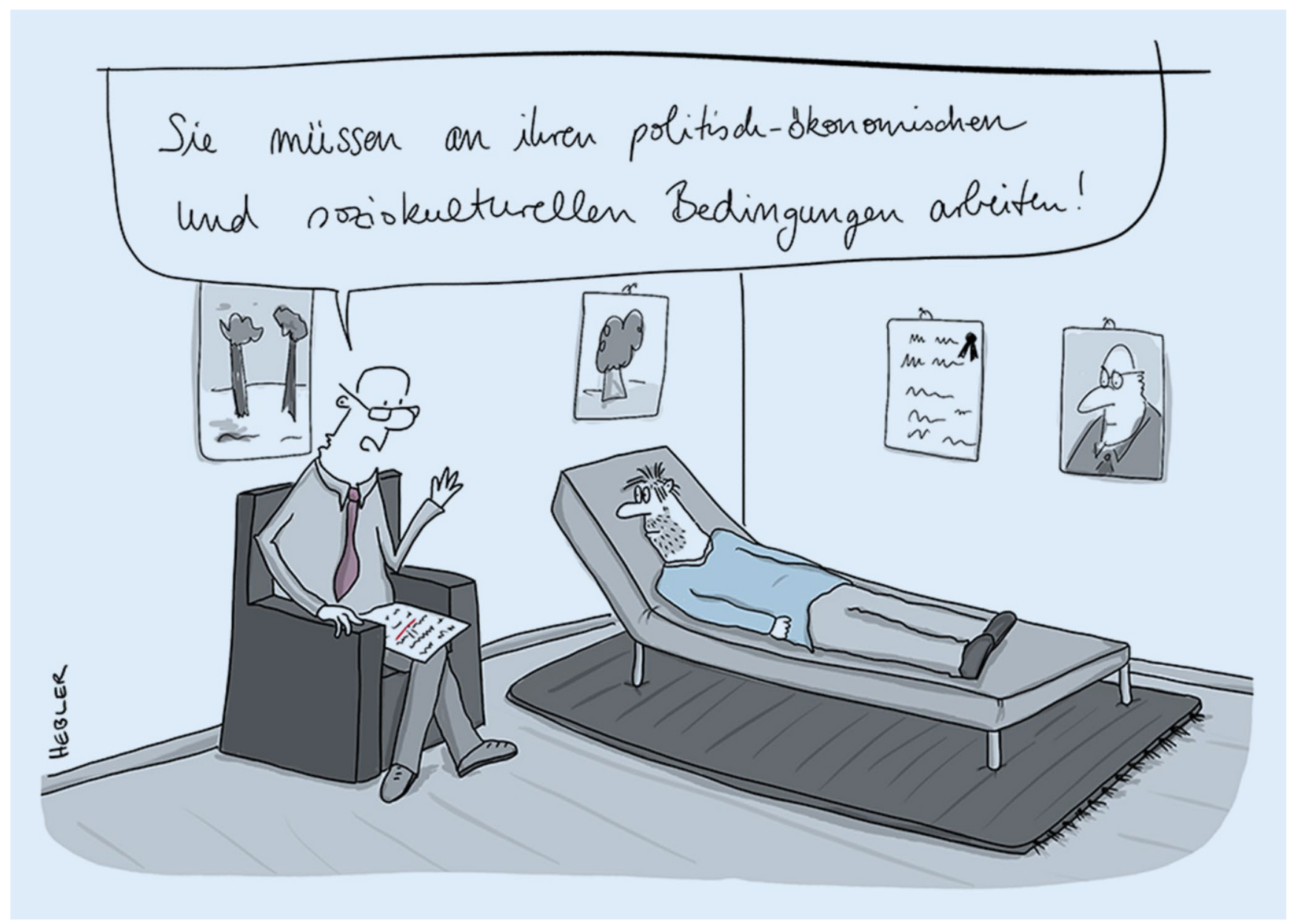

(c) Ruth Hebler

lung verstanden wird. Andererseits kommt es zu einer zunehmenden Vermittlung in den psychiatrischen und psychotherapeutischen Behandlungsapparat, wenn diese Angebote nicht mehr auszureichen scheinen. Die Selbstfürsorgekonzepte geraten spätestens dann in eine Krise, sobald die Bewältigungslage als „Krankheit“ codiert an das Gesundheitssystem adressiert und daraus ein individuelles "Scheitern“ entwickelt wird, verbunden mit verschiedenen Stigmatisierungsrisiken. Jedes Jahr steigen die Behandlungsfälle im Bereich psychischer Gesundheit, die Wartezeiten in den Psychotherapie-Praxen werden immer länger.

In kritischer Bezugnahme auf diese Entwicklung verweist auch Eva Illouz (2009) darauf, dass der therapeutische Diskurs in seiner Vielfalt zu einem der wichtigsten Codes geworden sei. Denn in dieser Perspektive werde das Leiden bzw. das fehlende Glück innerhalb einer Gesellschaft nicht mehr auf mögliche ökonomische, soziale oder politische Ursachen hin befragt, sondern zurückgeführt auf einen falschen Umgang mit den eigenen Gefühlen, der die moralische Pflicht zur Therapie nach sich ziehe (Illouz 2009; Cabanas und Illouz 2019).
Nicht zuletzt ist an diesen Entwicklungen die Soziale Arbeit als Theorie und Profession aktiv beteiligt, wenn sie etwa stigmatisierende Fallkategorien in ihren Hilfeplänen bildet oder „schwierige“ Klient_innen selbst in das Gesundheitssystem vermittelt, bis hin zur Anwendung von Profiling- und Verdinglichungsmechaniken in Modellen des Case-Managements zur „Herstellung des Klienten“ (vgl. Bittner 1981). Für moderne Formen der Sozialen Arbeit scheint der „neue Wein der selbstsorgenden und eigenverantwortlichen Arbeit am Selbst in den alten Schläuchen von Behandlung, Therapie und Individualisierung von gesellschaftliche Konflikten [...] attraktiv und professionalisierungsgeeignet" (Lutz 2016, S. 762) zu sein. Mit den folgenden Beiträgen möchten wir einige sowohl theoretische als auch praxisbezogene Schlaglichter auf den Diskurs werfen.

Die ersten beiden Beiträge thematisieren die Rolle von Sozialer Arbeit zwischen Medizin und Psychotherapie. Philipp Sperb skizziert diverse Fallstricke für Sozialarbeiter_innen, die eine suchttherapeutische Weiterbildung machen. Dabei wirft er Schlaglichter auf Strukturprobleme der suchttherapeutischen Ausbildung und 


\section{Durchblick: Therapeutisierung des Sozialen mit und in der Sozialen Arbeit}

Praxis, die durch deutungsmächtige, einseitig am Individuum orientierte Denk- und Handlungsmuster klinischer Disziplinen verschärft werden und die berufliche Identitätsbildung suchttherapeutischer Sozialer Arbeit auf der Grundlage eines konsequent bio-psycho-sozialen Modells erschweren. Helmut Schwehm betrachtet sein Thema „Soziale Arbeit im Spannungsfeld von Helfen, Heilen und Ver-Handeln “ unter der Frage, wie eine „Therapie des Sozialen“ möglich sein kann. Er sieht in der Philosophie und therapeutischen Methode des Psychodramas (Moreno) ein beträchtliches Potenzial, u. a. (lebens-)lagenspezifische Bedingungen in die Aufklärungspraxis Sozialer Arbeit einzubeziehen und so erkennbar einer einengenden Struktur einer „Therapeutisierung des Sozialen“ zu entgehen. Michael May erläutert in „Soziale Arbeit: Zwischen Reproduktion von Entfremdung durch Therapeutisierung und Perspektiven zu ihrer Überwindung " die Implikationen des verwendeten Marx'schen Entfremdungsbegriffs. Am Beispiel von enteignenden Umgang der Fachkräfte mit Gefühlen von Adressat_innen im sozialpsychiatrischen Handlungsfeld macht er anschaulich, wie auch Soziale Arbeit als Entfremdung reproduzierende Institution wirkmächtig wird. Eine Chance der Überwindung sieht er u. a. in einer genossenschaftlichen Organisation Sozialer Arbeit.

Im Kontext des sozialen Phänomens „Wohnungslosigkeit" macht Eckhard Rohrmann auf die zunehmende Verbreitung ontologisierender und psychopathologisierender Denk- und Handlungsmuster (auch) in der Sozialen Arbeit aufmerksam, die als eine - insbesondere für die Adressat_innen fatale - Wiederkehr eines „eigentlich“ überwunden geglaubten Problemverständnis gelesen werden müssen. Im Weiteren richten Katrin Heymann und Christian Kolbe den Blick auf sozialstaatliche Zurichtungsprozesse bei geflüchteten Menschen. Beispielhaft beleuchten sie die hier in den Vordergrund tretende Nützlichkeitspolitik mithilfe von Befunden aus dem Modellprojekt „Einwanderung gestalten " in NRW. Dabei wird u. a. deutlich, wie das aktuelle institutionelle Handeln auch Fragen nach der Integrationsfähigkeit und damit zusammenhängend nach Vermittlungshemmnissen wie „psychischen Auffälligkeiten" stellt, die die Eingliederung in den Arbeitsmarkt verhindern.

Wir hoffen, mit diesen Beiträgen den Diskursraum für dieses Thema wieder ein wenig geöffnet zu haben.
Funding. Open Access funding provided by Projekt DEAL.

Open Access. Dieser Artikel wird unter der Creative Commons Namensnennung 4.0 International Lizenz veröffentlicht, welche die Nutzung, Vervielfältigung, Bearbeitung, Verbreitung und Wiedergabe in jeglichem Medium und Format erlaubt, sofern Sie den/die ursprünglichen Autor(en) und die Quelle ordnungsgemäß nennen, einen Link zur Creative Commons Lizenz beifügen und angeben, $\mathrm{ob}$ Änderungen vorgenommen wurden.

Die in diesem Artikel enthaltenen Bilder und sonstiges Drittmaterial unterliegen ebenfalls der genannten Creative Commons Lizenz, sofern sich aus der Abbildungslegende nichts anderes ergibt. Sofern das betreffende Material nicht unter der genannten Creative Commons Lizenz steht und die betreffende Handlung nicht nach gesetzlichen Vorschriften erlaubt ist, ist für die oben aufgeführten Weiterverwendungen des Materials die Einwilligung des jeweiligen Rechteinhabers einzuholen.

Weitere Details zur Lizenz entnehmen Sie bitte der Lizenzinformation auf http://creativecommons.org/licenses/by/4.0/deed.de.

\section{Literatur}

Basaglia, F., \& Basaglia-Ongaro, F. (Hrsg.). (1980). Befriedungsverbrechen: über die Dienstbarkeit der Intellektuellen. Frankfurt a.M.: Europäische Verlagsanstalt.

Bittner, U. (1981). Wie der Klient „gemacht“ wird. In E. von Kardorff \& E. Koenen (Hrsg.), Psyche in schlechter Gesellschaft (S. 103-137). München: Urban \& Schwarzenberg.

Böhnisch, L. (1994). Gespaltene Normalität - Lebensbewältigung und Sozialpädagogik an den Grenzen der Wohlfahrtsgesellschaft. Weinheim, München: Juventa.

Cabanas, E., \& Illouz, E. (2019). Das Glücksdiktat und wie es unser Leben beherrscht. Frankfurt a.M.: Suhrkamp.

Durkheim, É. (1973). Der Selbstmord. Neuwied, Berlin: Luchterhand.

Durkheim, É. (1992). Über soziale Arbeitsteilung. Studie über die Organisation höherer Gesellschaften. Frankfurt a.M.: Suhrkamp.

Han, B.-C. (2014). Psychopolitik. Neoliberalismus und die neuen Machttechniken. Frankfurt a.M.: S. Fischer.

Hitzer, B. (2011). Die Therapeutisierung der Gefühle - eine Geschichte aus dem 20. Jahrhundert. Der Mensch, 42(43), 17-20.

Illouz, E. (2009). Die Errettung der modernen Seele. Therapien, Gefüble und die Kultur der Selbsthilfe. Frankfurt a.M.: Suhrkamp.

Lessenich, S. (2008). Die Neuerfindung des Sozialen. Der Sozialstaat im flexiblen Kapitalismus. Bielefeld: transcript.

Lutz, T. (2016). Therapeutisierung(en) und Pathologisierung(en) als Professionalisierungsmuster der Sozialen Arbeit: Responsibilisierung als Neuer Wein in Alten Schläuchen. In R. Anhorn \& M. Balzereit (Hrsg.), Handbuch Therapeutisierung und Soziale Arbeit (S. 749-766). Wiesbaden: Springer VS.

Maasen, S. (2011). Das beratene Selbst. Zur Genealogie der Therapeutisierung in den ,langen' Siebzigern: Eine Perspektivierung. In S. Maasen, et al. (Hrsg.), Das beratene Selbst. Zur Genealogie der Therapeutisierung in den, langen Siebziger (S. 7-33). Bielefeld: transcipt.

Merton, R. (1968). Social theory and social structure. New York: Free Press. 